\title{
Augmented Lagrangian method for optimizing non-orthogonal orbitals
}

\author{
Masato Sumita* \\ Center for Advanced Intelligence Project, RIKEN, \\ 1-4-1 Nihombashi, Chuo-ku, Tokyo, 103-0027, Japan. \\ International Center for Materials Nanoarchitectonics (WPI-MANA), \\ National Institute for Materials Science, 1-1 Namiki, Tsukuba, Ibaraki, 305-0044, Japan \\ Naruki Yoshikawa \\ Center for Advanced Intelligence Project, RIKEN, \\ 1-4-1 Nihombashi, Chuo-ku, Tokyo, 103-0027, Japan. \\ Department of Computer Science, University of Toronto, \\ 40 St. George Street, Toronto, Ontario M5S 2E4, Canada.
}

(Dated: November 16, 2020)

\begin{abstract}
We applied augmented Lagrangian method to optimize molecular wave function based on nonorthogonal orbitals (Spin coupled wave function; SCWF) for its grand-state energy. In contrast to the orthogonal-orbital-based electronic structure theory, SCWF includes spin eigenfunctions to satisfy the eigen states as the operator of the square of the spin. To obtain the ground-state energy of SCWF, therefore, it is necessary to optimize the orbital and the spin-coupling coefficients simultaneously. In this study, the spin-coupling and the orbital coefficients are optimized with the augmented Lagrangian method under the constrain of normality of the wave function. We employed this SCWF method to compute dissociative potential energy surfaces (PESs) of $\mathrm{H}_{2}, \mathrm{H}_{2}^{-}$, $\mathrm{He}_{2}^{+}$, and HLi. The obtained PESs by the SCWF method are close to these by full configuration interaction theory. These results indicate that the augmented Lagrangian method is effective to optimize SCWF.
\end{abstract}

\section{INTRODUCTION}

Recent electronic structure theories included in several simulation packages [1-3] had been developed based on the orthogonal orbitals, that is, molecular orbital (MO) method (Hartree-Fock approximation) [4]. However, the orthogonality between the orbitals is just a assumption and not an obligatory requirement to describe electronic structure on a molecule $[4,5]$. Owing to this assumption, we need to use unrestricted MO method to include the correlation between $\alpha$ and $\beta$ electrons in the same orbital. Hence, we always suffer from troubles like spin contamination [4], using these approximations for simulating chemical reactions or magnetic systems. To circumvent this problem, configuration interaction (CI) based theory represented as full $\mathrm{CI}$ and multi-configuration selfconsistent theory (MCSCF) had been developed [6].

In contrast to the much successful MO method, there are electronic structure theories that are developed to describe chemical reactions, which are conventionally known as valence bond (VB) methods [7-10] originated from the Heitler-London wave function [11]. In spite of the clarity of the VB method for explaining chemical reaction, the VB method had not been succeeded as a main tools for simulating chemical reactions owing to much demanding computational costs.

The VB method generally uses the non-orthogonal orbitals that require explicit spin eigenfunction. Hence,

\footnotetext{
* masato.sumita@riken.jp
}

it is a very formidable task to optimize the wave function in contrast to the MO method where minimization can be performed with iterative diagonalization used Hermitian of electronic Hamiltonian. Traditionally, the wave function based on the non-orthogonal orbitals are variationally optimized. In the case, analytical first-derivative to the electronic spatial orbital and spin (spin-coupling) coefficients is not enough to stabilize the energy but the analytical second-derivative [5, 12] to them is also necessary. In this study, we introduced the augmented Lagrangian method with the Broyden-Fletcher-Goldfarb-Shanno (BFGS) algorithm [13] using numerical gradient to optimize these coefficients for the energies and validated its accuracy by comparing the full CI potential energy surface (PES).

The augmented Lagrangian method or method of multipliers $[14,15]$ is an algorithm to solve constrained optimization problems. It is used in quantum chemistry for reducing the computational cost results in computational acceleration under the constrain of idempotent of density matrix $[16,17]$. Here, we applied this algorithm to optimize the orbital and spin-coupled coefficients for the energy of wave function based on non-orthogonal orbitals.

\section{NON-ORTHOGONAL ORBITALS SYSTEM}

\section{A. Spin coupled wave function}

Wave function of $N$ electrons based on non-orthogonal orbitals are called spin coupled wave function (SCWF) 
proposed by Gerratt and expected to show much stability rather than molecular orbital [5]. The SCWF are written in the following form,

$$
\Psi_{S M}=\sum_{k}^{f_{s}} C_{S k} \sqrt{N !} \mathscr{A}\left(\phi_{1} \phi_{2} \ldots \phi_{N} \Theta_{S M k}\right)
$$

$C_{S k}$ is the spin-coupling coefficient. $\mathscr{A}$ is a well-known anti-symmetrizer imposing the anti-symmetry of Fermion to the wave function;

$$
\mathscr{A}=\frac{1}{N !} \sum_{P} \epsilon_{P} P .
$$

$\phi_{i}(i=1,2, \ldots N)$ is familiar linear combination of basis functions $\left(\chi_{j}\right)$ with coefficient $C_{i j}$ and the normalized orbitals but non-orthogonal to each other;

$$
\begin{gathered}
\phi_{i}=\sum_{j} C_{i j} \chi_{j}, \\
\left\langle\phi_{i} \mid \phi_{k}\right\rangle=\Delta_{i k} .
\end{gathered}
$$

$\mathscr{A}$ includes permutations $(P)$ all over $N$ electrons with its parity $\epsilon_{P}$. $P$ operates on both spatial and spin parts. $\Theta_{S M k}$ is an $N$-electron spin function. The number of the degeneracy of spin eigenfunction is represented by $f_{s}$. When the number of spin is $S$, the number of independent spin functions with the $S_{z}$ eigenvalue $M=S$ is given by,

$$
f_{s}=\left(\begin{array}{c}
N \\
N / 2-S
\end{array}\right)-\left(\begin{array}{c}
N \\
N / 2-S-1
\end{array}\right) .
$$

Hence, eq. (1) indicates that the linear combination of the independent spin functions. Although there are several ways to construct spin eigenfunctions [18, 19], we employed the branching-diagram method to construct the orthonormal spin systems because the operation of a permutation on a spin function can be represented by the linear combination of independent spin functions with the coefficient of matrix elements $U_{l k}(P)$ of symmetric group;

$$
P \Theta_{k}=\epsilon_{P} \sum_{l}^{f s} U_{l k}(P) \Theta_{l} .
$$

\section{B. Energy of SCWF}

The Hamiltonian of $N$ electrons is defined by,

$$
\begin{aligned}
\mathscr{H} & =\sum_{\mu}^{N} h_{\mu}+\frac{1}{2} \sum_{\mu, \nu} g_{\mu, \nu} \\
& =\mathbf{h}+\frac{1}{2} \mathbf{g} .
\end{aligned}
$$

here, $h_{\mu}$ and $g_{\mu \nu}(\mu, \nu=1,2, \ldots N)$ are the one electron and two electron operators respectively. Briefly, they are described in bold style as $\mathbf{h}$ and $\mathbf{g}$. Electronic energy of the wave function of eq. (1) can be obtain as the expectation value of the Hamiltonian. Thus, the ground state energy $(E)$ is obtained by minimizing

$$
E(\mathbf{C}, \mathbf{C s})=\frac{\left\langle\Psi_{S M}|\mathscr{H}| \Psi_{S M}\right\rangle}{\left\langle\Psi_{S M} \mid \Psi_{S M}\right\rangle}
$$

The numerator of eq. (8) can be deformed by substituting $\Psi_{S M}$ with eq. (1).

$$
\left.E_{M S}(\mathbf{C}, \mathbf{C s})\right)=\left\langle\Psi_{S M}|\mathscr{H}| \Psi_{S M}\right\rangle=\frac{1}{N !} \sum_{k, l}^{f_{s}} C_{S k} C_{S l} \sum_{P R}\left\langle P\left(\phi_{1} \phi_{2} \ldots \phi_{N}\right)|\mathscr{H}| R\left(\phi_{1} \phi_{2} \ldots \phi_{N}\right)\right\rangle\left\langle P \Theta_{k} \mid R \Theta_{l}\right\rangle
$$

Hereafter, we introduce the notation, $\mu_{1} \mu_{2} \ldots \mu_{N}$ and $\nu_{1} \nu_{2} \ldots \nu_{N}$ as electron arrangements after operating the permutation $P$ and $R$ to $\phi_{1} \phi_{2} \ldots \phi_{N}$. As will be shown letter, because we use SymPy package [20] to decompose a permutation to elementary transpositions, the following formulated representation is employed as the original electron arrangement is always $\phi_{1} \phi_{2} \ldots \phi_{N}$. Hence, the spatial parts of eq. (9) can be described as the following,

$$
\begin{aligned}
& \sum_{P R}\left\langle\mu_{1} \mu_{2} \ldots \mu_{N}|\mathscr{H}| \nu_{1} \nu_{2} \ldots \nu_{N}\right\rangle=\sum_{P R}\left\{\left\langle\mu_{1} \mu_{2} \ldots \mu_{N}|\mathbf{h}| \nu_{1} \nu_{2} \ldots \nu_{N}\right\rangle+\frac{1}{2}\left\langle\mu_{1} \mu_{2} \ldots \mu_{N}|\mathbf{g}| \nu_{1} \nu_{2} \ldots \nu_{N}\right\rangle\right\} \\
& =\sum_{P R}\left\{\left\langle\mu_{1}|\mathbf{h}| \nu_{1}\right\rangle\left\langle\mu_{2} \mid \nu_{2}\right\rangle \ldots\left\langle\mu_{N} \mid \nu_{N}\right\rangle+\frac{1}{2}\left\langle\mu_{1} \mu_{2}|\mathbf{g}| \nu_{1} \nu_{2}\right\rangle . .\left\langle\mu_{N} \mid \nu_{N}\right\rangle\right\} . \\
& \because P=\left(\begin{array}{cccc}
\phi_{1} & \phi_{2} & \ldots & \phi_{N} \\
\mu_{1} & \mu_{2} & \ldots & \mu_{N}
\end{array}\right), R=\left(\begin{array}{llll}
\phi_{1} & \phi_{2} & \ldots & \phi_{N} \\
\nu_{1} & \nu_{2} & \ldots & \nu_{N}
\end{array}\right)
\end{aligned}
$$


On the other hand, spin parts of eq. (9) can be deformed as the following by using eq. (6) and the orthonormality of the spin eigenfunctions.

$$
\begin{aligned}
\left\langle P \Theta_{k} \mid R \Theta_{l}\right\rangle & =\epsilon_{P} \epsilon_{R}\left\langle\sum_{m} U_{m k}(P) \Theta_{m} \mid \sum_{n} U_{n l}(R) \Theta_{n}\right\rangle \\
& =\epsilon_{P} \epsilon_{R} \sum_{m, n} U_{k m}(P) U_{n l}(R)\left\langle\Theta_{m} \mid \Theta_{n}\right\rangle \\
& =\epsilon_{P} \epsilon_{R} U_{k l}(P R) \quad \because\left\langle\Theta_{m} \mid \Theta_{n}\right\rangle=\delta_{m n}
\end{aligned}
$$

For spatial parts, because the one-electron integral for any electrons $(1,2, \ldots, N)$ has the same value and the two-electron integral for any electron-pairs $(N(N-1))$ is also in the summation over all permutations $(P, R)$, we can obtain the following form,

$$
\begin{aligned}
\left.E_{M S}(\mathbf{C}, \mathbf{C s})\right) & =\frac{1}{N !} \sum_{k, l}^{f_{s}} C_{S k} C_{S l} \sum_{P R} \epsilon_{P} \epsilon_{R} U_{k l}(P R)\left\{\left\langle\mu_{1}|\mathbf{h}| \nu_{1}\right\rangle\left\langle\mu_{2} \mid \nu_{2}\right\rangle \ldots\left\langle\mu_{N} \mid \nu_{N}\right\rangle+\frac{1}{2}\left\langle\mu_{1} \mu_{2}|\mathbf{g}| \nu_{1} \nu_{2}\right\rangle . .\left\langle\mu_{N} \mid \nu_{N}\right\rangle\right\} \\
& =\sum_{k, l}^{f_{s}} C_{S k} C_{S l} \sum_{P R} \epsilon_{P} \epsilon_{R} U_{k l}(P R)\left\{\frac{1}{(N-1) !}\left\langle\mu_{1}|h| \nu_{1}\right\rangle\left\langle\mu_{2} \mid \nu_{2}\right\rangle \ldots\left\langle\mu_{N} \mid \nu_{N}\right\rangle\right. \\
& \left.+\frac{1}{2(N-2) !}\left\langle\mu_{1} \mu_{2}|g| \nu_{1} \nu_{2}\right\rangle . .\left\langle\mu_{N} \mid \nu_{N}\right\rangle\right\} .
\end{aligned}
$$

Therefore, $N ! \times N$ ! terms of both one-electron and two-electron integrals should be computed. Here, we dropped the indices of electron $\mu$ and $\nu$ for $h$ and $g$.

For the computation of the denominator of eq. (8), we just compute $N$ ! overlap integrals because of the similarity, fixing the electronic arrangement of the left side in the bracket to the original $\phi_{1} \phi_{2} \ldots \phi_{N}$, i.e.

$$
\begin{aligned}
\Delta(\mathbf{C}, \mathbf{C s}) & =\left\langle\Psi_{S M} \mid \Psi_{S M}\right\rangle \\
& =\sum_{k, l}^{f_{s}} C_{S k} C_{S l} \sum_{P R} \epsilon_{P} \epsilon_{R} U_{k l}(P R) \frac{1}{N !}\left\langle\mu_{1} \mid \nu_{1}\right\rangle\left\langle\mu_{2} \mid \nu_{2}\right\rangle \ldots\left\langle\mu_{N} \mid \nu_{N}\right\rangle \\
& =\sum_{k, l}^{f_{s}} C_{S k} C_{S l} \sum_{R} \epsilon_{R} U_{k l}(R)\left\langle\phi_{1} \mid \nu_{1}\right\rangle\left\langle\phi_{2} \mid \nu_{2}\right\rangle \ldots\left\langle\phi_{N} \mid \nu_{N}\right\rangle .
\end{aligned}
$$

Gerratt et al. [12, 21] obtained the energy of SCWF, optimizing $\mathbf{C s}$ and orbital expansion coefficients $(\mathbf{C})$ for the energy of eq. (8). On the other hand, McWeeny [22] suggested optimizing (12) under the constraint of normality of SCWF represented in eq. (13) $(\Delta(\mathbf{C}, \mathbf{C s})=$ $\left.\left\langle\Phi_{S M} \mid \Phi_{S M}\right\rangle=1\right)$. We employed the latter method to optimize the energy of SCWF with the augmented Lagrangian method in this work.

\section{Augmented Lagrangian method}

A constrained optimization problem with $m$ equality constraints is formulated as follows:

$$
\begin{array}{ll}
\operatorname{minimize} & f(\mathbf{x}) \\
\text { subject to } & c_{i}(\mathbf{x})=0 \quad(1 \leq i \leq m)
\end{array}
$$

The augmented Lagrangian method solves this constrained optimization problem by repeatedly solving an unconstrained optimization problem. In the $k$-th step of the algorithm, the unconstrained optimization problem

$$
\text { minimize } f(\mathbf{x})+\mu_{k} \sum_{i=1}^{m} c_{i}(\mathbf{x})^{2}+\sum_{i=1}^{m} \lambda_{k, i} c_{i}(\mathbf{x}),
$$

is solved. $\quad \lambda_{k, i}$ and $\mu_{k}$ are initialized as $\lambda_{1, i}=0$, and $\mu_{1}=1$. In each step, they are updated as $\lambda_{k+1, i}=\lambda_{k, i}+$ $2 \mu c_{i}\left(\mathbf{x}_{k}\right)$ and $\mu_{k+1}=2 \mu_{k}$, where $\mathbf{x}_{k}$ is the solution to the $k$-th step. This process is repeated until the conversion criteria $\left|c_{i}\left(\mathbf{x}_{k}\right)\right|<\varepsilon$ is satisfied for all $1 \leq i \leq m$.

In our method, we solve a constrained optimization problem where the objective function $f(\mathbf{x})$ is the numerator of eq. (8), $E_{M S}(\mathbf{C}, \mathbf{C s})$ represented in eq. (12), and the constraint $c_{i}(\mathbf{x})=0$ is the normality constraint $\Delta(\mathbf{C}, \mathbf{C s})-1=0$.

\section{COMPUTATIONAL DETAILS}

All molecular integrals were obtained via PySCF package [23]. Since any permutations can be represented 


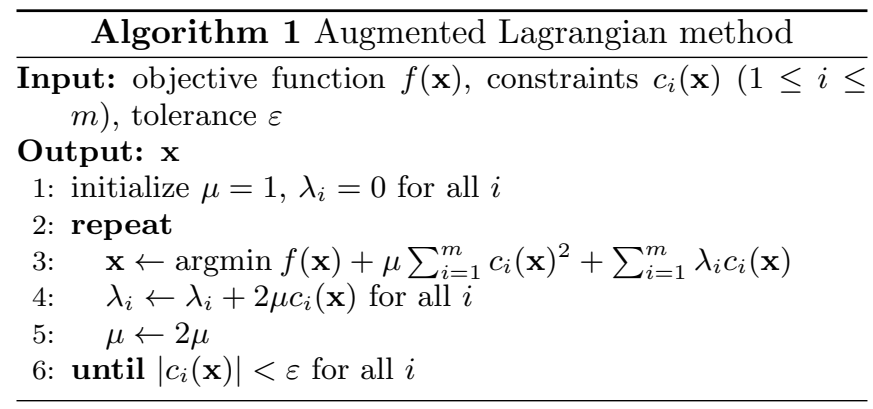

by the multiply of elementary transpositions, the coefficients of matrix elements $U_{l k}(P)$ of symmetric group are prepared only for the elementary transpositions in an $N$-electron system. To decompose a permutation to the product of transpositions, we used SymPy package [20]. To reduce the computational costs, we employed a minimum basis set, STO-3G. Initial values of $\mathbf{C}_{\mathbf{s}}$ and $\mathbf{C}$ are set random numbers but $\mathbf{C}_{\mathbf{s}}$ is normalized and $\mathbf{C}$ is set to the values so as to satisfy eq. (4) before the SCWF energy computation.

In our implementation of the augmented Lagrangian method (Algorithm 1), the unconstrained optimization problem was solved by BFGS [13] implemented in SciPy [24], and we set $\varepsilon=10^{-8}$. The gradient for BFGS was estimated using 2-point finite difference estimation with SciPy's default setting.

\section{RESULTS AND DISCUSSION}

We used SCWF optimized by the augmented Lagrangian method to describe the PESs of $\mathrm{H}_{2}, \mathrm{H}_{2}^{-}, \mathrm{He}_{2}^{+}$, and $\mathrm{HLi}$ as the function of their internuclear distances and compared them with the PESs at the full CI level. As the non-orthogonal orbital is the open shell, SCWF is good at describing the dissociation of chemical bonds. Furthermore, it is easy to get the spin states through the spin-coupled coefficients. Here, we validate the SCWF with the augmented Lagrangian method from the qualitative and quantitative viewpoints.

\section{A. $\mathrm{H}_{2}$, two electron system}

In two electron systems, the spin eigenfunction for the singlet state is given as the following,

$$
\Theta_{0}=\frac{1}{\sqrt{2}}\left\{\alpha_{1} \beta_{2}-\beta_{1} \alpha_{2}\right\},
$$

where the subscript numbers indicate the indices of electrons. The number of degeneracy of spin eigenfunction is 1. Hence, $\mathbf{C}_{\mathbf{S}}$ is a constant, 1 , and the orbital coefficients are the values to be optimized. As an example of two electron systems, we computed the bond breaking process of

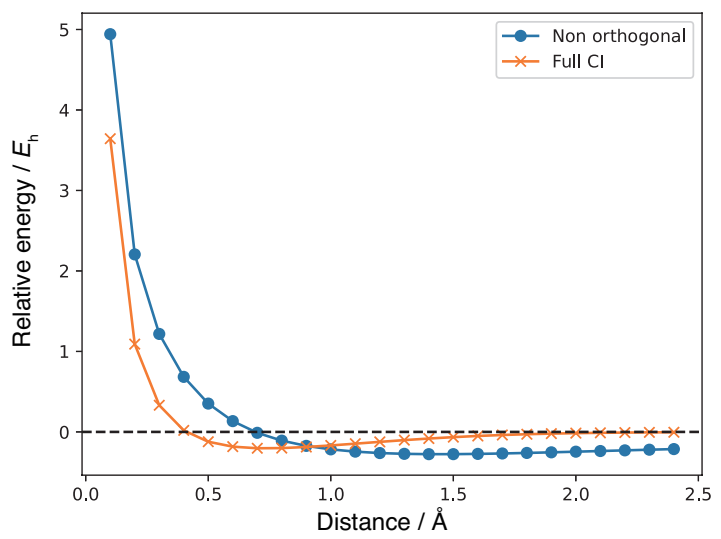

FIG. 1. PESs of $\mathrm{H}_{2}$ as the function of internuclear distance computed by full-CI and SCWF. Relative energies are computed to the dissociated limitation at each computational level.

hydrogen molecule, $\mathrm{H}_{2}$. The PESs as the function of internuclear distance at the full CI and SCWF with augmented Lagradian method are shown Fig. 1. The PES by SCWF shows the minimum at which the internuclear distance is $1.4 \AA$ and lies at $6.16 \mathrm{eV}$ lower than dissociate limitation, which is much lower than experimental results $(4.75 \mathrm{eV})$. This result indicates the over optimization of SCWF by the augmented Lagradian method. Overestimation of the equilibrium would be attributed to the lack of constrained condition. Unfortunately, we could not figure out the suitable condition to elevate the accuracy except for replacing the object function to eq. (8), which brings unstability to PESs of the other test systems. In the longer range, however, the relative energy of SCWF is stabilized to the degree of full-CI.

The non-orthogonal orbitals of electron 1 and 2 are shown in Fig. 2. Although both symmetric orbitals are almost localized on respective nucleus, the overlap integral with them is computed as the order of $10^{-4}$, that seems to be small but enough to stabilize the $\mathrm{H}_{2}$ system. Since eq. (17) indicates the coupling between electron 1 and 2, we can confirm the appearance of the valence bond between hydrogen atoms.

\section{B. $\mathrm{H}_{2}^{-}, \mathrm{He}_{2}^{+}$, three electron systems}

More than three electron systems, spin eigenfunctions degenerated with each others. In the three electron doublet systems, the following two functions are degenerated,

$$
\begin{aligned}
& \Theta_{0}=\frac{1}{\sqrt{6}}\left\{2 \alpha_{1} \alpha_{2} \beta_{3}-\alpha_{1} \beta_{2} \alpha_{3}-\beta_{1} \alpha_{2} \alpha_{3}\right\}, \\
& \Theta_{1}=\frac{1}{\sqrt{2}}\left\{\alpha_{1} \beta_{2} \alpha_{3}-\beta_{1} \alpha_{2} \alpha_{3}\right\} .
\end{aligned}
$$

In the $\mathrm{H}_{2}^{-}$system, the equilibrium internuclear dis- 


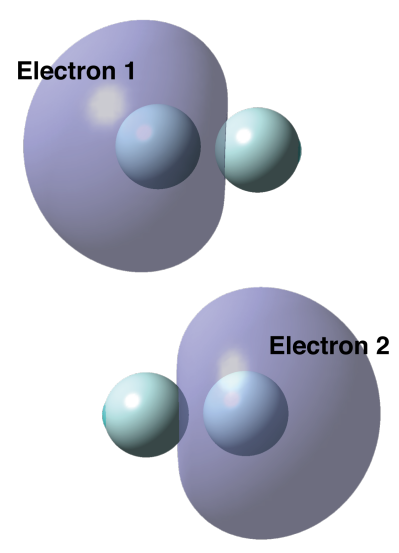

FIG. 2. non-orthogonal orbitals for electron 1 and 2 of $\mathrm{H}_{2}$ at which internuclear distance is $0.7 \AA$.

tance and the binding energy are reported as $0.8 \AA$ and $1.7 \mathrm{eV}$ respectively [25]. As shown in Fig. 3, the SCWF qualitatively reproduced the PES at the full CI level. However, the internuclear distance and the binding energy are overestimated, that is, the minimum lies at 5.68 $\mathrm{eV}$ around 1.1 $\AA$. Optimized $\mathbf{C}_{\mathbf{S}}$ as the function of the internuclear distance is shown in the bottom of Fig. 3 . From the internuclear distance longer than $0.5 \AA$, the dominant spin configuration is eq. (18) and its occupancy is over $90 \%$. This function indicates that the spin couplings of electron 3 with 1 and 2. At the internuclear distance of $1.1 \AA$, the orbital of electron 1 mainly localized on one nucleus and that of electron 3 mainly localized on the other nucleus as shown in Fig. 4. Hence, the coupling between 1 and 3 indicates the valence bond between nucleus. On the other hand, the orbital of electron 2 is delocalized on both nucleus with the anti-bond character as like the LUMO of $\mathrm{H}_{2}$ as shown in Fig. 4. Clearly, electron 2 is the radical electron that is only coupled with electron 3 . This result indicates the qualitatively correct description that the character of $\mathrm{H}-\mathrm{H}$ valence bond is deduced by coupling with the radical electron. However, the orbital symmetry is not conserved. Then the overestimation of the binding energy is owing to the symmetry breaking [26]. From the internuclear distance shorter than $0.5 \AA$, the spin configuration eq. (19) is dominant. Because eq. (19) represents that the spin coupling between electron 1 and 2, this spin configuration just contribute the destabilization of the system because bonding character of electron 1 is offset by anti-bonding character of electron 2.

On the other hand, the SCWF failed to reproduce the existence of the minimum in the $\mathrm{He}_{2}^{+}$system though the PES at the SCWF level is close to that at the full CI level as shown in Fig. 5. In similar way to the $\mathrm{H}_{2}^{-}$system, dominant spin configuration is eq. (18) whose occupation is over $90 \%$ in the region of longer internuclear distance than $0.6 \AA$. The non-orthognal orbital of electron 1, 2, and 3 are shown in Fig. 6. In contrast to the $\mathrm{H}_{2}$ and $\mathrm{H}_{2}^{-}$systems, orbitals are symmetric and all elec-
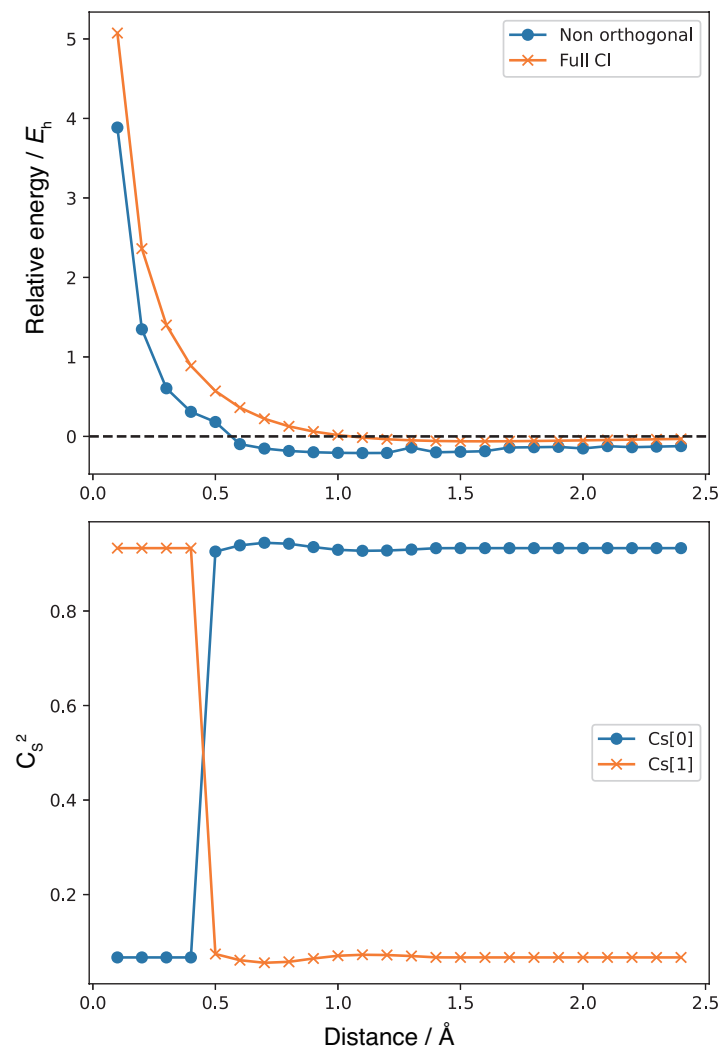

FIG. 3. PESs of $\mathrm{H}_{2}^{-}$as the function of internuclear distance computed by full-CI and SCWF (top). Relative energies are computed to the dissociated limitation at each computational level. Square of spin-coupling coefficients along with the top PES of $\mathrm{H}_{2}^{-}$(bottom). Cs[0] and $\mathrm{Cs}[1]$ are the coefficients of eq. (18) and (19) respectively.

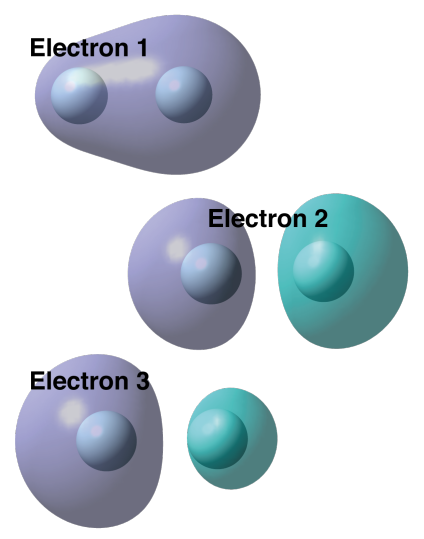

FIG. 4. non-orthogonal orbitals for electron 1, 2, and 3 of $\mathrm{H}_{2}^{-}$ at which internuclear distance is $1.1 \AA$.

trons delocalized on both nucleus but only electron 2 has anti-bonding character. Because eq. (18) indicates the coupling of electron 3 with electron 1 and 2, the system is mainly stabilized by the coupling of electron 3 with electron 1. However, in the region of shorter internuclear distance than $0.5 \AA$, the dominance of eq. (18) collapses 

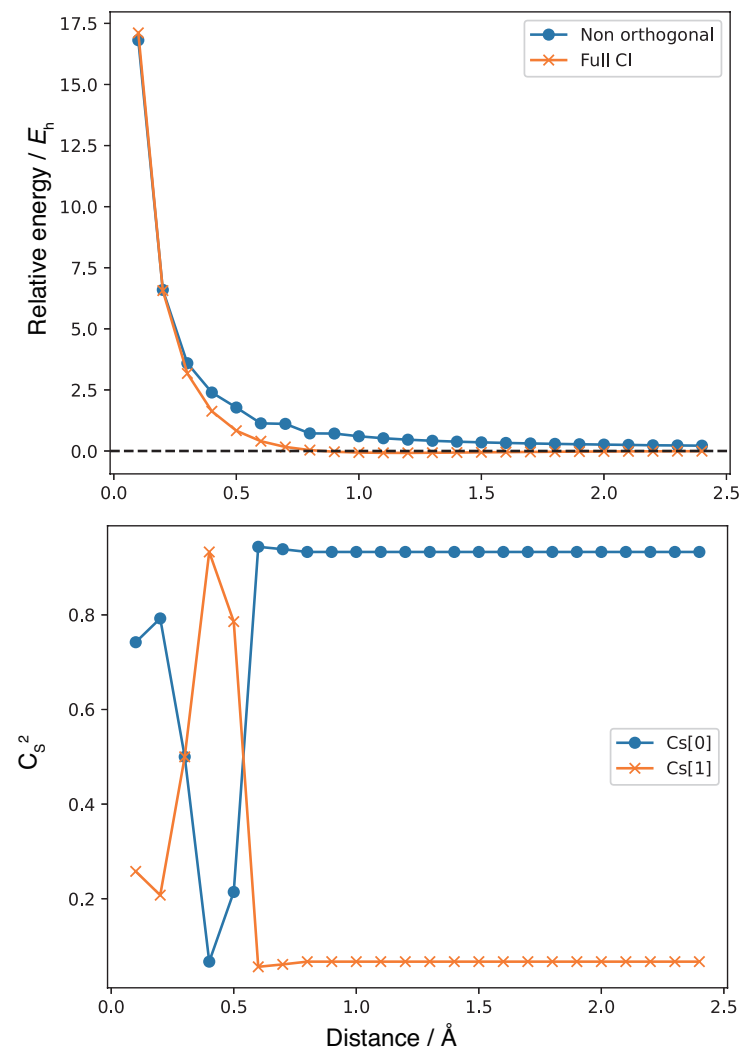

FIG. 5. PESs of $\mathrm{He}_{2}^{+}$as the function of internuclear distance computed by full-CI and SCWF (top). Relative energies are computed to the dissociated limitation at each computational level. Square of spin-coupling coefficients along with the top PES of $\mathrm{H}_{2}^{-}$(bottom). Cs[0] and $\mathrm{Cs}[1]$ are the coefficients of eq. (18) and eq. (19) respectively.

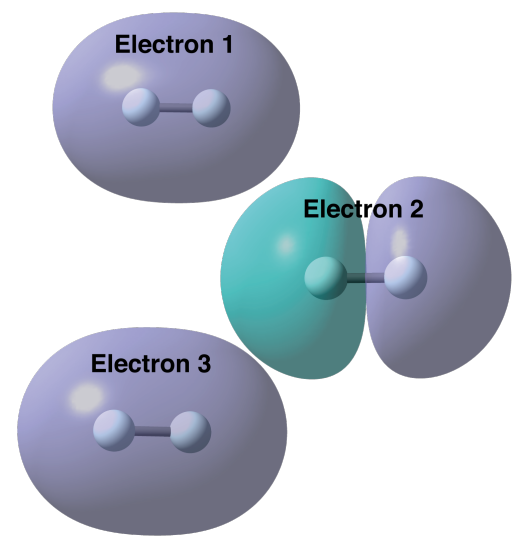

FIG. 6. non-orthogonal orbitals for electron 1, 2, and 3 of $\mathrm{He}_{2}^{+}$at which internuclear distance is $1.1 \AA$.

and the spin configuration of eq. (19) mixes suddenly not gradually. Therefore, in the shorter region, crossing between electronic states may be involved in this drastic change of spin configuration states.

\section{HLi, four electron system}

Finally, we have checked heteronuclear diatomic molecule, HLi, it is expected to show ionic (heterolytic) dissociation to $\mathrm{H}^{-}$and $\mathrm{Li}^{+}$. The PES as the function of internuclear distance is shown in the top of Fig. 7 . We found the existence of a minimum at $1.1 \AA$, that is underestimated in comparison with the experimental one $(1.596 \AA)$ and the dissociation energy is overestimated as $4.25 \mathrm{eV}$ (experimentaly $2.498 \mathrm{eV}$ ) [27]. However, qualitative tendency of dissociative PES agrees with that of full CI. Furthermore, ionic character of this system agree with the qualitative speculation.

In this system, four electrons and six primitive basis set functions are involved and the following two spin eigenfunctions are degenerated.

$$
\begin{aligned}
\Theta_{0} & =\frac{1}{\sqrt{12}}\left\{2 \alpha_{1} \alpha_{2} \beta_{3} \beta_{4}-\alpha_{1} \beta_{2} \alpha_{3} \beta_{4}-\beta_{1} \alpha_{2} \alpha_{3} \beta_{4}\right. \\
& \left.-\alpha_{1} \beta_{2} \beta_{3} \alpha_{4}-\beta_{1} \alpha_{2} \beta_{3} \alpha_{4}+2 \beta_{1} \beta_{2} \alpha_{3} \alpha_{4}\right\}, \\
\Theta_{1} & =\frac{1}{2}\left\{\alpha_{1} \beta_{2} \alpha_{3} \beta_{4}-\beta_{1} \alpha_{2} \alpha_{3} \beta_{4}\right. \\
& \left.-\alpha_{1} \beta_{2} \beta_{3} \alpha_{4}+\beta_{1} \alpha_{2} \beta_{3} \alpha_{4}\right\} .
\end{aligned}
$$

Eq. (20) indicates the following electron couplings, pairs of 2-4, 1-3 electrons, and pairs of 1-4, 2-3 electrons. On the other hand, eq. (21) indicates the electron coupling, pairs of 1-2, 3-4 electrons. As shown in Fig. 8, electron 1 and 4 are the 1s orbital of Li slightly combined with 1 s orbital of $\mathrm{H}$, and electron 2 and 3 mainly attributes to the $1 \mathrm{~s}$ orbital of $\mathrm{H}$ combined with the $1 \mathrm{~s}$ of Li. Hence, we can conclude that $\mathrm{H}$ negatively charged and Li positively. Furthermore, the pairs of 1-2/3-4 electrons indicate the interaction like a valence bond between 1s of $\mathrm{Li}$ and $1 \mathrm{~s}$ of $\mathrm{H}$ but its spin configuration is always inferior in the whole PES shown in Fig. 7. On the other hand, the dominant spin configuration includes two electron coupling style. One is the pairs of 1-4 and 2-3, which clearly indicate the interaction within $1 \mathrm{~s}$ of $\mathrm{Li}$ and $1 \mathrm{~s}$ of $\mathrm{H}$ respectively. The other is the pairs of $1-3$ and 2-4, which indicate valence bond like interaction between $\mathrm{H}^{-}$ and $\mathrm{Li}^{+}$. Therefore, we can conclude that not only ionic but also valence bond interaction also contribute the stabilization of the HLi system, which is widely believed as a representative ionic interaction system.

\section{CONCLUSION}

In this work, the augmented Lagrangian method is employed to optimize the orbital coefficients and spincoupling coefficients of the spin-coupled wave function (SCWF) for its energy. The application of the SCWF to compute dissociative PES of $\mathrm{H}_{2}, \mathrm{H}_{2}^{-}, \mathrm{He}_{2}^{+}$, and $\mathrm{HLi}$ shows that the PESs qualitatively close to those of the full-CI level without depending on the nature of (homolytic/heterolytic) dissociation. Therefore, we can con- 

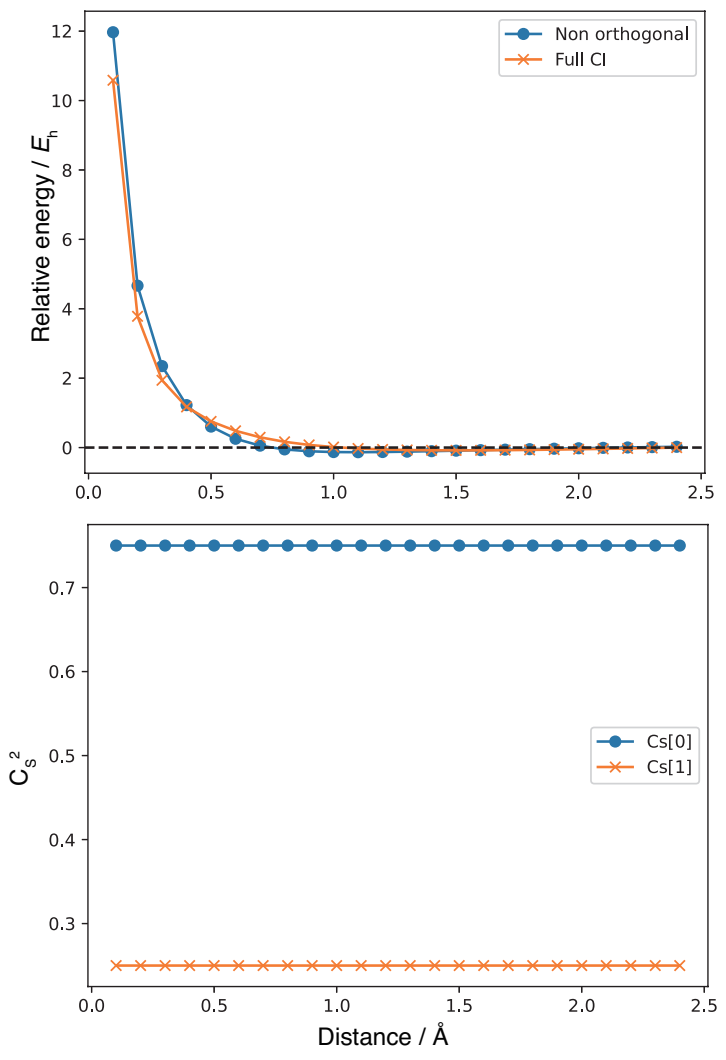

FIG. 7. PESs of HLi as the function of H-Li bond length computed by full-CI and SCWF (top). Relative energies are computed to the dissociated limitation at each computational level. Square of spin-coupling coefficients along with the top PES of $\mathrm{H}_{2}^{-}$(bottom). Cs[0] and $\mathrm{Cs}[1]$ are the coefficients of eq. (20) and (21) respectively.

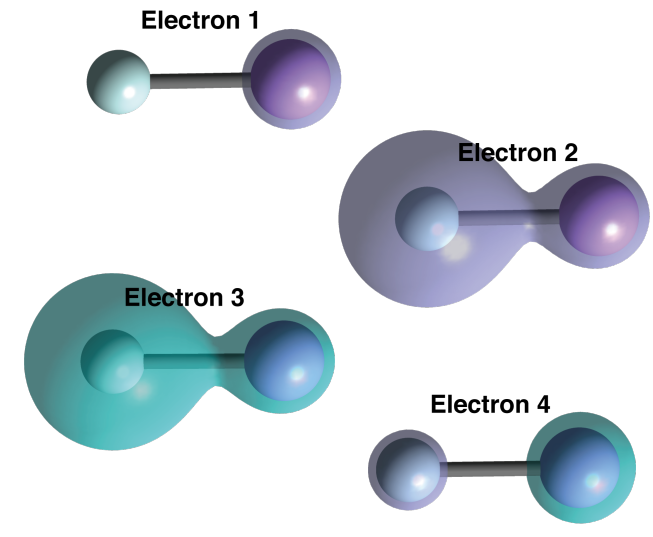

FIG. 8. non-orthogonal orbitals for electron 1, 2, 3, and 3 of $\mathrm{HLi}$ at which internuclear distance is $1.6 \AA$.

clude that the augmented Lagrangian method is effective to optimize the energy of SCWF. However, quantitative structure at the minimum of each system did not agree with those at the full-CI level or experimental results. Particularly, as shown in $\mathrm{H}_{2}^{-}$, even for SCWF, it is impossible to avoid the symmetry breaking that is also a formidable problem for any molecular orbital based method. This means that SCWF would also need the state average method to avoid this problem.

Unfortunately, the SCWF presented here is not for quantitative discussion. For quantitative discussion, SCWF probably requires suitable basis sets. At the present study, we did not check basis set dependence because of heavy computational cost. Our current code requires 1.4 hours to compute the SCWF energy by optimizing 24 orbital coefficients and 2 spin-coupling coefficients because the processes of constructing spin eigenfucntion and counting electron pairs are time consuming. Developing applicable SCWF algorithm to more large system is our future work.

Because SCWF includes spin eigenfunctions that have information about the electron coupling, SCWF makes it possible to discuss the qualitative chemical reaction process and the electronic structure at equilibrium from the different viewpoint of molecular orbital methods. Although the spin eigenfunction does not involve the energy of a molecule directly, it is important to describe chemical reactions and magnetic systems [10]. Recent several computation models for complex material systems like Li-ion batteries interface SumitaPolymer, oxidation on GaN surface systems [28] indicate the importance of the treatment of spin state in the computational model. Additionally, correct treatment of spin state is of importance to analyze the systems that include singlet and triplet oxygen molecules and singlet diradical systems [29, 30]. Therefore, the methods that ensure a spin eigen state of target systems, like SCWF as shown in this study and CASSCF [6] would become important.

\section{ACKNOWLEDGEMENT}

The computations in this work were carried out on the supercomputer center of RAIDEN of AIP (RIKEN). 
[1] M. J. Frisch, G. W. Trucks, H. B. Schlegel, G. E. Scuseria, M. A. Robb, J. R. Cheeseman, G. Scalmani, V. Barone, G. A. Petersson, H. Nakatsuji, X. Li, M. Caricato, A. V. Marenich, J. Bloino, B. G. Janesko, R. Gomperts, B. Mennucci, H. P. Hratchian, J. V. Ortiz, A. F. Izmaylov, J. L. Sonnenberg, D. WilliamsYoung, F. Ding, F. Lipparini, F. Egidi, J. Goings, B. Peng, A. Petrone, T. Henderson, D. Ranasinghe, V. G. Zakrzewski, J. Gao, N. Rega, G. Zheng, W. Liang, M. Hada, M. Ehara, K. Toyota, R. Fukuda, J. Hasegawa, M. Ishida, T. Nakajima, Y. Honda, O. Kitao, H. Nakai, T. Vreven, K. Throssell, J. A. Montgomery, Jr., J. E. Peralta, F. Ogliaro, M. J. Bearpark, J. J. Heyd, E. N. Brothers, K. N. Kudin, V. N. Staroverov, T. A. Keith, R. Kobayashi, J. Normand, K. Raghavachari, A. P. Rendell, J. C. Burant, S. S. Iyengar, J. Tomasi, M. Cossi, J. M. Millam, M. Klene, C. Adamo, R. Cammi, J. W. Ochterski, R. L. Martin, K. Morokuma, O. Farkas, J. B. Foresman, and D. J. Fox, Gaussian ${ }^{\sim} 16$ Revision C.01 (2016), gaussian Inc. Wallingford CT.

[2] G. M. J. Barca, C. Bertoni, L. Carrington, D. Datta, N. De Silva, J. E. Deustua, D. G. Fedorov, J. R. Gour, A. O. Gunina, E. Guidez, T. Harville, S. Irle, J. Ivanic, K. Kowalski, S. S. Leang, H. Li, W. Li, J. J. Lutz, I. Magoulas, J. Mato, V. Mironov, H. Nakata, B. Q. Pham, P. Piecuch, D. Poole, S. R. Pruitt, A. P. Rendell, L. B. Roskop, K. Ruedenberg, T. Sattasathuchana, M. W. Schmidt, J. Shen, L. Slipchenko, M. Sosonkina, V. Sundriyal, A. Tiwari, J. L. Galvez Vallejo, B. Westheimer, M. Wloch, P. Xu, F. Zahariev, and M. S. Gordon, Recent developments in the general atomic and molecular electronic structure system, The Journal of Chemical Physics 152, 154102 (2020).

[3] E. Aprá, E. J. Bylaska, W. A. de Jong, N. Govind, K. Kowalski, T. P. Straatsma, M. Valiev, H. J. J. van Dam, Y. Alexeev, J. Anchell, V. Anisimov, F. W. Aquino, R. Atta-Fynn, J. Autschbach, N. P. Bauman, J. C. Becca, D. E. Bernholdt, K. Bhaskaran-Nair, S. Bogatko, P. Borowski, J. Boschen, J. Brabec, A. Bruner, E. Cauët, Y. Chen, G. N. Chuev, C. J. Cramer, J. Daily, M. J. O. Deegan, T. H. Dunning, M. Dupuis, K. G. Dyall, G. I. Fann, S. A. Fischer, A. Fonari, H. Früchtl, L. Gagliardi, J. Garza, N. Gawande, S. Ghosh, K. Glaesemann, A. W. Götz, J. Hammond, V. Helms, E. D. Hermes, K. Hirao, S. Hirata, M. Jacquelin, L. Jensen, B. G. Johnson, H. Jónsson, R. A. Kendall, M. Klemm, R. Kobayashi, V. Konkov, S. Krishnamoorthy, M. Krishnan, Z. Lin, R. D. Lins, R. J. Littlefield, A. J. Logsdail, K. Lopata, W. Ma, A. V. Marenich, J. Martin del Campo, D. Mejia-Rodriguez, J. E. Moore, J. M. Mullin, T. Nakajima, D. R. Nascimento, J. A. Nichols, P. J. Nichols, J. Nieplocha, A. Otero-de-la Roza, B. Palmer, A. Panyala, T. Pirojsirikul, B. Peng, R. Peverati, J. Pittner, L. Pollack, R. M. Richard, P. Sadayappan, G. C. Schatz, W. A. Shelton, D. W. Silverstein, D. M. A. Smith, T. A. Soares, D. Song, M. Swart, H. L. Taylor, G. S. Thomas, V. Tipparaju, D. G. Truhlar, K. Tsemekhman, T. Van Voorhis, A. Vázquez-Mayagoitia, P. Verma, O. Villa, A. Vishnu, K. D. Vogiatzis, D. Wang, J. H. Weare, M. J. Williamson, T. L. Windus, K. Woliński, A. T. Wong, Q. Wu, C. Yang, Q. Yu, M. Zacharias,
Z. Zhang, Y. Zhao, and R. J. Harrison, Nwchem: Past, present, and future, The Journal of Chemical Physics 152, 184102 (2020).

[4] A. Szabo and N. S. Ostlund, Modern Quantum Chemistry (Dover Publications, Inc., 1989).

[5] J. Gerratt and W. N. Lipscomb, Proc. Natl. Acad. Sci. (USA) 59, 332 (1968).

[6] B. O. Roos and P. R. Taylor, Chem. Phys. 48, 157 (1980).

[7] D. L. Cooper, M. Raimondi, and J. Gerratt, Chem. Rev. 91, 929 (1991).

[8] J. Gerratt, D. L. Cooper, P. B. Karadakov, and M. Raimondi, Chem. Soc. Rev. 26, 87 (1997).

[9] G. A. Gallup, Valence Bond Methods: Theory and applications (Cambridge University Press, 2002).

[10] D. L. Cooper, ed., Valence Bonc Theory, Theoretical and computational, chemistry, Vol. 10 (Elsevier, 2002).

[11] W. Heitler and F. London, Z. Physik 44, 455 (1927).

[12] N. C. Pyper and J. Gerratt, Proc. R. Soc. Lond. A 355, 407 (1977).

[13] J. Nocedal and S. Wright, Numerical optimization (Springer Science \& Business Media, 2006).

[14] M. R. Hestenes, Multiplier and gradient methods, Journal of optimization theory and applications 4, 303 (1969).

[15] M. J. Powell, A method for nonlinear constraints in minimization problems, Optimization , 283 (1969).

[16] S. Adhikari and R. Baer, Augmented lagrangian method for order-n electronic structure, The Journal of chemical physics 115, 11 (2001).

[17] A. Staszczak, M. Stoitsov, A. Baran, and W. Nazarewicz, Augmented lagrangian method for constrained nuclear density functional theory, The European Physical Journal A 46, 85 (2010).

[18] R. Pauncz, Spin Eigenfunctions Construction and Use (Springer Science+Business Media New York, 1979).

[19] R. Pauncz, The Construction of Spin Eigenfunctions An Exercise Book (Kluwer Academic/Plenum Publishers, 2000).

[20] A. Meurer, C. P. Smith, M. Paprocki, O. Čertík, S. B. Kirpichev, M. Rocklin, A. Kumar, S. Ivanov, J. K. Moore, S. Singh, T. Rathnayake, S. Vig, B. E. Granger, R. P. Muller, F. Bonazzi, H. Gupta, S. Vats, F. Johansson, F. Pedregosa, M. J. Curry, A. R. Terrel, v. Roučka, A. Saboo, I. Fernando, S. Kulal, R. Cimrman, and A. Scopatz, Sympy: symbolic computing in python, PeerJ Computer Science 3, e103 (2017).

[21] J. Gerratt and M. Raimondi, Proc. R. Soc. Lond. A 371, 525 (1980).

[22] R. McWeeny, Methods of Molecular Quantum Mechanics (Academic Press, 2001).

[23] Q. Sun, T. C. Berkelbach, N. S. Blunt, G. H. Booth, S. Guo, Z. Li, J. Liu, J. D. McClain, E. R. Sayfutyarova, S. Sharma, S. Wouters, and G. K.-L. Chan, Pyscf: the python-based simulations of chemistry framework, Wiley Interdisciplinary Reviews: Computational Molecular Science 8, e1340 (2018).

[24] P. Virtanen, R. Gommers, T. E. Oliphant, M. Haberland, T. Reddy, D. Cournapeau, E. Burovski, P. Peterson, W. Weckesser, J. Bright, S. J. van der Walt, M. Brett, J. Wilson, K. J. Millman, N. Mayorov, A. R. J. Nelson, E. Jones, R. Kern, E. Larson, C. J. Carey, İ. Polat, Y. Feng, E. W. Moore, J. VanderPlas, D. Laxalde, 
J. Perktold, R. Cimrman, I. Henriksen, E. A. Quintero, C. R. Harris, A. M. Archibald, A. H. Ribeiro, F. Pedregosa, P. van Mulbregt, and SciPy 1.0 Contributors, SciPy 1.0: Fundamental Algorithms for Scientific Computing in Python, Nature Methods 17, 261 (2020).

[25] J. P. Lowe, Quantum Chemistry (Academic Press Inc., 1993).

[26] E. R. Davisdon and W. T. Borden, J. Phys. Chem. 87, 4783 (1983).

[27] Y. C. Chan, D. R. Harding, W. C. Stwalley, and C. R. Vidal, J. Phys. Chem. 85, 2436 (1986).
[28] M. Sumiya, M. Sumita, Y. Asai, R. Tamura, A. Uedono, and A. Yoshigoe, J. Chem. Phys. C XX, XX (2020).

[29] M. Abe, J. Ye, and M. Mishima, Chem. Soc. Rev. 41, 3808 (2012).

[30] M. Sumita and K. Morihashi, J. Phys. Chem. A 119, 876 (2015).

[31] M. Sumita, Y. Tanaka, and T. Ohno, J. Chem. Phys. C 121, 9698-9704 (2017). 